First CT using Medipix3 and the MARS-CT-3 spectral scanner

This article has been downloaded from IOPscience. Please scroll down to see the full text article.

2011 JINST 6 C01095

(http://iopscience.iop.org/1748-0221/6/01/C01095)

View the table of contents for this issue, or go to the journal homepage for more

Download details:

IP Address: 137.138.124.233

The article was downloaded on 27/07/2012 at 08:23

Please note that terms and conditions apply. 


\section{First CT using Medipix3 and the MARS-CT-3 spectral scanner}

M.F. Walsh, ${ }^{a}{ }^{1}$ A.M.T. Opie, ${ }^{b}$ J.P. Ronaldson, ${ }^{a}$ R.M.N. Doesburg, ${ }^{c}$ S.J. Nik, ${ }^{c}$ J.L. Mohr, ${ }^{c}$ R. Ballabriga, ${ }^{d}$ A.P.H. Butler ${ }^{a}$ and P.H. Butler ${ }^{c}$

${ }^{a}$ Centre of Bioengineering, University of Otago Christchurch, Riccarton Ave, Christchurch, New Zealand

${ }^{b}$ Department of Electrical Engineering, University of Canterbury, Ilam Rd, Christchurch, New Zealand

${ }^{c}$ Department of Physics, University of Canterbury, Ilam Rd, Christchurch, New Zealand

${ }^{d}$ Organisation Européenne pour la Recherche Nucléaire (CERN), Geneva, Switzerland

E-mail: triorph@gmail.com

ABSTRACT: The MARS research group has created a new version of their scanner for taking improved spectral CT datasets. This version of the scanner (MARS-CT-3) has taken the first Medipix3 CT images of a phantom. MARS-CT-3 incorporates a new gantry, new x-ray sources and the new MARS readout board, as well as the ability to connect gas lines to the specimen.

The new gantry has improved mechanical rigidity and can perform scans faster. Magnification can be controlled by moving the detector and the $\mathrm{x}$-ray source independently. The brighter $\mathrm{x}$-ray source means images can be taken six times faster. Gas lines allow the user to control various environmental factors inside the scanner, such as temperature, or deliver oxygen and anaesthetics, providing the ability to do a full spectroscopic CT scan of a live sedated biological specimen, such as a mouse. The new MARS readout is able to read from all current chips from the Medipix family, has faster image downloading, and the use of up to six Medipix detectors in parallel on the same chip carrier. The use of Medipix 3 chips allows for compensation of charge sharing via Charge Summing Mode.

KeYwORDS: X-ray detectors; Computerized Tomography (CT) and Computed Radiography (CR); Solid state detectors

\footnotetext{
${ }^{1}$ Corresponding author.
} 


\section{Contents}

1 Introduction 1

2 The MARS-CT-3 2

3 Medipix3 and charge summing mode 2

4 Results and conclusion 3

\section{Introduction}

Computed Tomography (CT) is a technique for constructing 2D cross sections and 3D images from a series of $2 \mathrm{D}$ projections. It has become a valuable tool in medical diagnosis and research. However x-ray images have poor soft tissue contrast. A common technique to enhance x-ray images is to use contrast agents (pharmaceuticals) to enhance features of interest [1]. Although this helps, it can be difficult to distinguish between the contrast agent and other natural high contrast objects (e.g. bone, etc.)

The attenuation of photons through a material varies non-linearly based on the energy of the photons [2]. This variation enables CT to discriminate soft tissues from each other, and has been shown effective at discriminating various radiographic contrast agents [3].

The Medipix family of detectors is used to measure the attenuation in various energy bins. Techniques such as PCA [4] or SOM [5] can be used to create a spectral x-ray image where colour is assigned to different materials. Figure 1 shows a spectral $\mathrm{x}$-ray image of a mouse. The bone in the image appears as blue, while the soft tissue appears as a darker colour.

The MARS research group are working on custom CT scanners which can house Medipix detectors. This paper discusses the latest version of this custom CT scanner. Preliminary results are also presented.

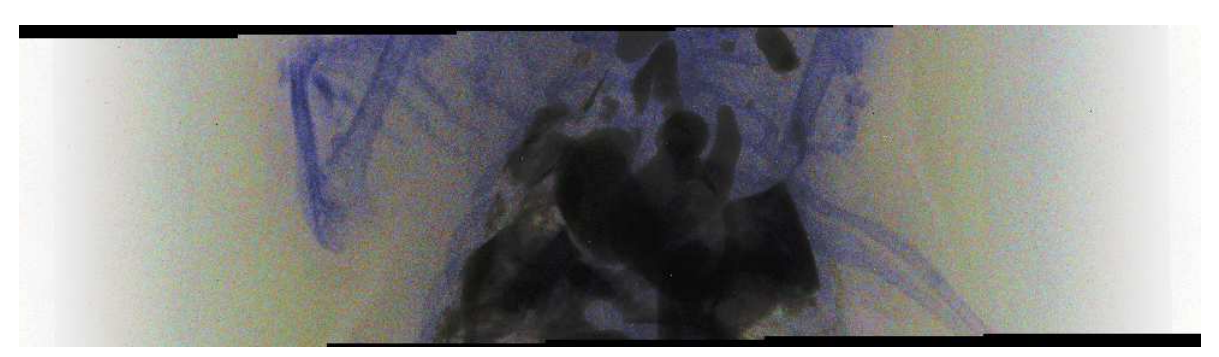

Figure 1. A spectral x-ray projection of a mouse, taken with Medipix3. 


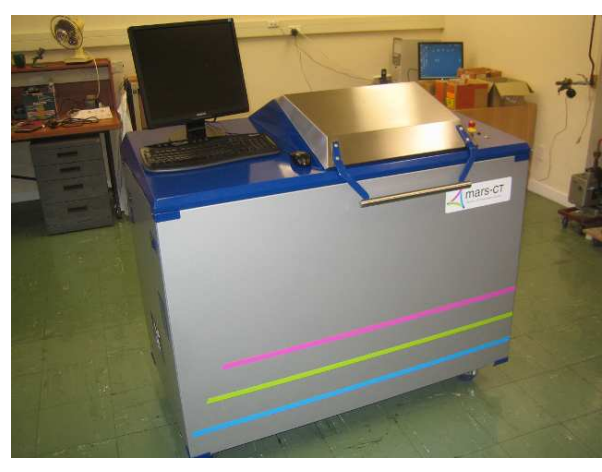

(a) The MARS-CT-3 scanner

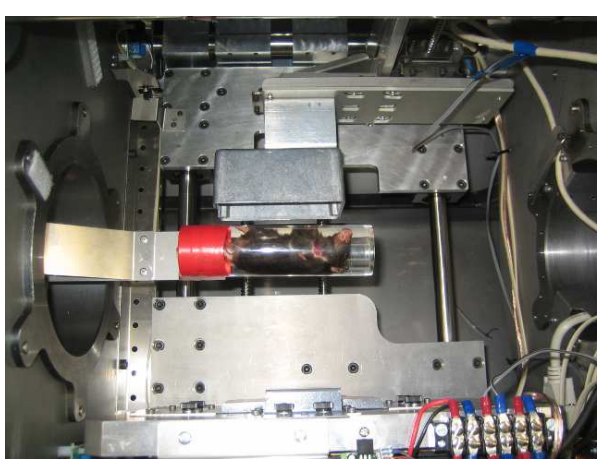

(b) The internal gantry

Figure 2. The MARS-CT-3 scanner and its internal gantry.

\section{The MARS-CT-3}

The new MARS-CT-3 scanner has several improvements. The more important advances are an improved gantry design, a new x-ray source, the addition of gas lines, and the use of the MARS readout board. Figure 2(a) shows the complete MARS-CT-3 system.

The new gantry design offers smoother movements than the old design and is capable of moving faster. The rotational skew of the detector is only out by an error of one pixel, the old scanner was out by an error of four pixels. Scans are accomplished by stopping the gantry rotation at every image angle. This takes 2.5 seconds per rotation stop, and approximately 15 minutes for 360 stops. The ability to translate the x-ray source and camera independently means magnification can be accurately controlled to be within 1.45 and 2.64. Figure 2(b) shows the internal gantry of the MARS-CT-3 scanner.

The scanner can hold two new $\mathrm{x}$-ray sources. They are capable of $\mathrm{kVp}$ values up to $80 \mathrm{kV}$ and $120 \mathrm{kV}$ and currents of up to $1000 \mu \mathrm{A}$ and $350 \mu \mathrm{A}$ respectively [6]. The previous source was only capable of $75 \mathrm{kVp}$ and $150 \mu \mathrm{A}$ [7]. This choice of $\mathrm{x}$-ray sources enables either the ability to capture images six times faster, or to extend the energy range up to $120 \mathrm{keV}$ photons.

The latest MARS readout board supports a variety of Medipix chips, including the Medipix3 and MXR. Using a multi-chip carrier, up to six Medipix chips can be installed, with up to four chips currently being used. Image acquisition over a large field of view is feasible with a parallel connection of multiple MARS readouts. The MARS readout board has the potential for the imaging speed to be as high as a $50 \mathrm{~Hz}$ frame rate.

\section{Medipix 3 and charge summing mode}

Medipix3 is the latest in the line of Medipix spectroscopic x-ray detectors. It is capable of operating at 8 different threshold counters per image with $110 \mu \mathrm{m} \times 110 \mu \mathrm{m}$ pixels in a $128 \times 128$ array, or 2 threshold counters at $55 \mu \mathrm{m} \times 55 \mu \mathrm{m}$ in a $256 \times 256$ array [8].

One of the issues found in the previous Medipix chips was charge sharing [9], where charge carriers released by a single highly energetic photon are collected by several pixels, with a varying percentage of the total charge deposited in each pixel. The previous chips detected these as separate photon counts, and create a large number of incorrect photon counts in the low energy ranges. The original photon count of interest will also be detected with less charge. 


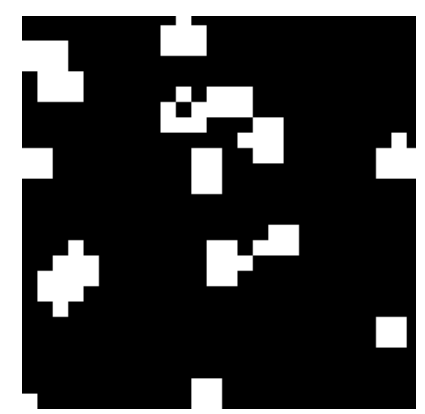

(a) Normal Image, 7000 counts over full range. Approximately 13 clusters are shown.

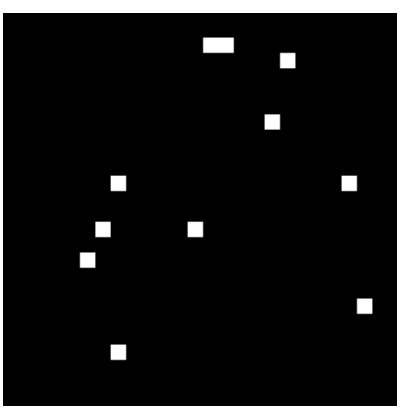

(b) Charge Summing Mode Image, 700 counts over full range.

11 pixels are showing counts.

Figure 3. $26 \times 26$ pixel section of alpha particles to demonstrate Charge Summing Mode. The Charge Summing Mode image is closer to the true photon count than the Normal.

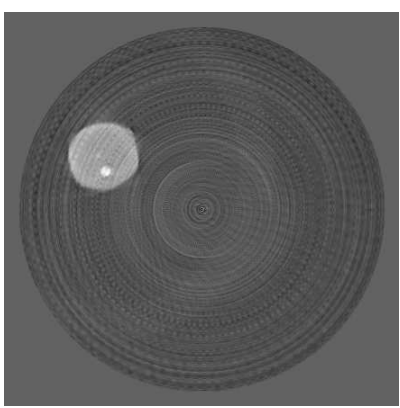

(a) Wire through high contrast spheroid

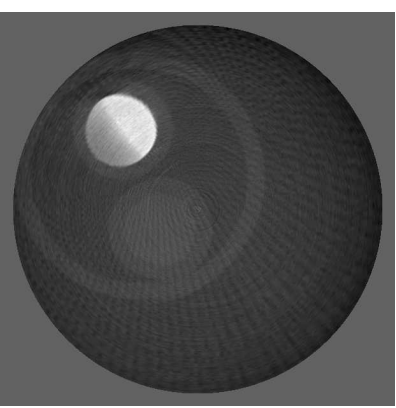

(b) Two syringes. Bright syringe contains iodine, the other water

Figure 4. First CT results with Medipix3. Taken with the MARS-CT-3 scanner.

The Medipix3 chip has a Charge Summing Mode that allows for charge sharing compensation. In Charge Summing Mode, adjacent pixels with simultaneous deposition communicate to sum up the charges from these adjacent pixels and assign the total to the pixel with the greatest original charge.

\section{Results and conclusion}

Figure 3 shows two images taken with Medipix3. These demonstrate the benefit of charge summing. In the left figure alpha particles deposit their charge in clusters of 8-20 pixels. In the right figure the charge summing allocates the interaction of a $3 \times 3$ cluster to a single pixel. This is reflected by the Charge Summing Mode image having approximately one ninth the counts of the Normal image. For regular x-ray use, charge sharing will create clusters of $2 \times 2$ or less.

Figure 4 contains the first CT images obtained with Medipix 3 and MARS-CT-3 scanner. Each image is the sum of all the slices. The iodine in the right image shows a variation in intensity because there is a bubble caught in the volume of interest. 
We have successfully built a micro-CT scanner for biomedical imaging. The scanner is more flexible than previous versions in that it allows control of magnification, brighter and higher energy $\mathrm{x}$-ray sources, a range of Medipix detector types and larger areas of Medipix chips. This allows for future work on obtaining spectral CT images with Medipix3.

\section{References}

[1] S. Yu and A.D. Watson, Metal-Based x-ray contrast media, Chem. Rev. 99 (1999) 2353.

[2] J. Giersch and J. Durst, Monte carlo simulations in x-ray imaging, Nucl. Instrum. Meth. A 591 (2008) 300.

[3] N.G. Anderson et al., Spectroscopic (multi-energy) CT distinguishes iodine and barium contrast material in MICE, Europ. Radiol. 20 (2010) 2126.

[4] B. Moore, Principal component analysis in linear systems: Controllability, observability, and model reduction, IEEE Trans. Autom. Control 26 (1981) 17.

[5] T. Kohonen, The self-organizing map, Proc. IEEE 78 (1990) 1464.

[6] I. Source-Ray, Model SB-80-1K (Doc. M-SB801K-DI, Rev 1), Installation/Operation Manual, http://www.sourceray.com/manuals/enduser/M-SB-80-1K-DI_REV_1.pdf.

[7] T. Scientific, Kevex PXS11-150-75, 75kV Minifocus X-Ray Source, http://tinyurl.com/2d2rtqu.

[8] R. Ballabriga, M. Campbell, E. Heijne, X. Llopart and L. Tlustos, The medipix3 prototype, a pixel readout chip working in single photon counting mode with improved spectrometric performance, IEEE Nucl. Sci. Symp. Conf. Rec. (2006) 3557.

[9] H. Zeller et al., Charge sharing between pixels in the spectral medipix $2 x$-ray detector, in Image and Vision Computing New Zealand, 2009. IVCNZ '09, 24th International Conference (2009) pg. 363-366. 